\title{
Lung function trajectories using different reference equations in a birth cohort study up to the age of 20 years
}

\author{
To the Editor:
}

New spirometry reference values from the Global Lung Function Initiative (GLI) and the LUNOKID (Lungenfunktions-Normalwerte bei Kindern in Deutschland [Lung function Normal Values of Children in Germany]) study were published in 2012 and 2013, respectively, using a nonlinear model for lung function trajectories [1-3]. There is a lack of empirical testing of these new equations and reference values in longitudinal cohort studies.

In 2015, Stanojevic et al. [4] applied the GLI reference equations to data from two longitudinal cystic fibrosis registries. They were able to demonstrate that the use of GLI equations showed a steady rate of decline in forced expiratory volume in $1 \mathrm{~s}$ (FEV1) in childhood cystic fibrosis, whereas traditional equations, in part, showed periods of accelerated decline in adolescence [4]. So far, this has been the only longitudinal study; evaluations including healthy or asthmatic subjects are lacking.

To determine how lung function parameters vary over time, long-term observational (birth) cohort studies with multiple time points of respiratory health assessments are superior to clinical trials examining interventions for lung or other diseases. In the present study, we aimed to evaluate the longitudinal trajectories of lung function in a birth cohort up to the age of 20 years using different reference equations. We also aimed to assess the fit of different equations to the German cohort by separately analysing data for nonasthmatic and asthmatic subgroups from early childhood to young adulthood.

Data were used from the Multicentre Allergy Study (MAS). This German birth cohort study was initiated in 1990 with 1314 newborns recruited from six obstetrics departments [5], including lung function measurements at four different time points between 7 and 20 years. We computed z-scores for FEV1, forced vital capacity (FVC) and FEV1/FVC for each follow-up by using the equations from ZAPLETAL et al. [6], LUNOKID [2,3] and GLI [1] for children aged 7, 10 and 13 years. For young adults at 20 years, we used equations of GLI and the European Community for Steel and Coal (ECSC) [7]. Traditionally, we combined ZaPLETAL et al. [6] and ECSC reference data for longitudinal graphing. Cases with more than two missing data in the follow-up were excluded from the analyses. We applied criteria for asthma definition at the last follow-up at 20 years. As suggested and implemented by several European population-based birth cohort consortia from the Global Allergy and Asthma European Network, Environmental Health Risks in European Birth Cohorts, and Mechanisms of the Development of Allergy initiatives, asthma was defined by a positive response to at least two of the following three questions $[8,9]$ : a parent- or self-reported physician's diagnosis of asthma (ever), current wheezing (in last 12 months) and/or current asthma medication (in the previous 12 months). Data were available for 700 subjects; 74 of these were classified as asthmatics at the age of 20 years. Nonlinear cubic spline curves were used to model lung function trajectories by different reference systems. $95 \%$ confidence intervals were calculated to indicate differences between different time points. Maximum decreases in $\mathrm{z}$-scores were assessed by calculating the difference between the highest and lowest mean $\mathrm{z}$-score of three follow-up time points (7, 10 and 13 years). Single $\mathrm{z}$-scores of nonasthmatic subjects were interpreted as sufficient fit to each reference equation in case of a value between -0.5 and 0.5 [10].

@ERSpublications

Lung function trajectories using different reference equations in a birth cohort up to the age of 20 years show a drop in FEV1 and FVC of both asthmatic and nonasthmatic subjects at the age of 13 years regardless of which reference equations were used http://ow.ly/hxGJ30ktUYE

Cite this article as: Lex C, Reuter M, Schuster A, et al. Lung function trajectories using different reference equations in a birth cohort study up to the age of 20 years. Eur Respir J 2018; 52: 1800364 [https://doi.org/ 10.1183/13993003.00364-2018]. 
Interpreting the spline curves for FEV1 and FVC of nonasthmatic participants, mean z-scores decreased from the ages of 7 and 10 years to the age of 13 years (puberty), and increased from the age of 13 to the age of 20 years. The maximum mean decreases of FEV1 in z-scores were 0.54 (GLI), 0.19 (LUNOKID) and 0.34 (ZAPLETAL et al. [6]). The maximum mean decreases of FVC in z-scores were 0.52 (GLI), 0.19 (LUNOKID) and 0.23 (ZAPlETAL et al. [6]) (figure 1). This drop at 13 years was equally seen in asthmatic and nonasthmatic subjects. Spline curves of asthmatic patients were below those of nonasthmatic participants. The drop in lung function trajectory at 13 years was also observed when asthma diagnosis was made at the age of 13 years instead of the age of 20 years.

Spline curves for FEV1/FVC only decreased when applying the ZAPLETAL et al. [6]/ECSC equations. However, there was no drop in puberty, but instead in early school age (7-10 years) and in young adulthood. In contrast, spline curves for FEV1/FVC using GLI or LUNOKID equations were stable during childhood and adolescence.

The FEV1 drop at 13 years was clearly more pronounced among males than females. However, the difference was just significant for ZAPLETAL et al. [6] and not for GLI. Additionally, the gap at 13 years between boys and girls was also observed for FEV1/FVC.

Analysing single FEV1 and FVC $\mathrm{z}$-scores showed that healthy subjects mostly had values between -0.5 and 0.5 . In contrast FEV1/FVC values were all $>0.5$ in this group.

To our knowledge, this is the first longitudinal study applying GLI and other reference equations on multiple lung function data in a birth cohort. We found a $\mathrm{z}$-score decrease in FEV1 and FVC at the age of 13 years, and a subsequent increase between the age of 13 and 20 years, considering that lung function of young teenagers can be difficult to interpret. Lung function trajectories were equal for both asthmatic and nonasthmatic subjects.
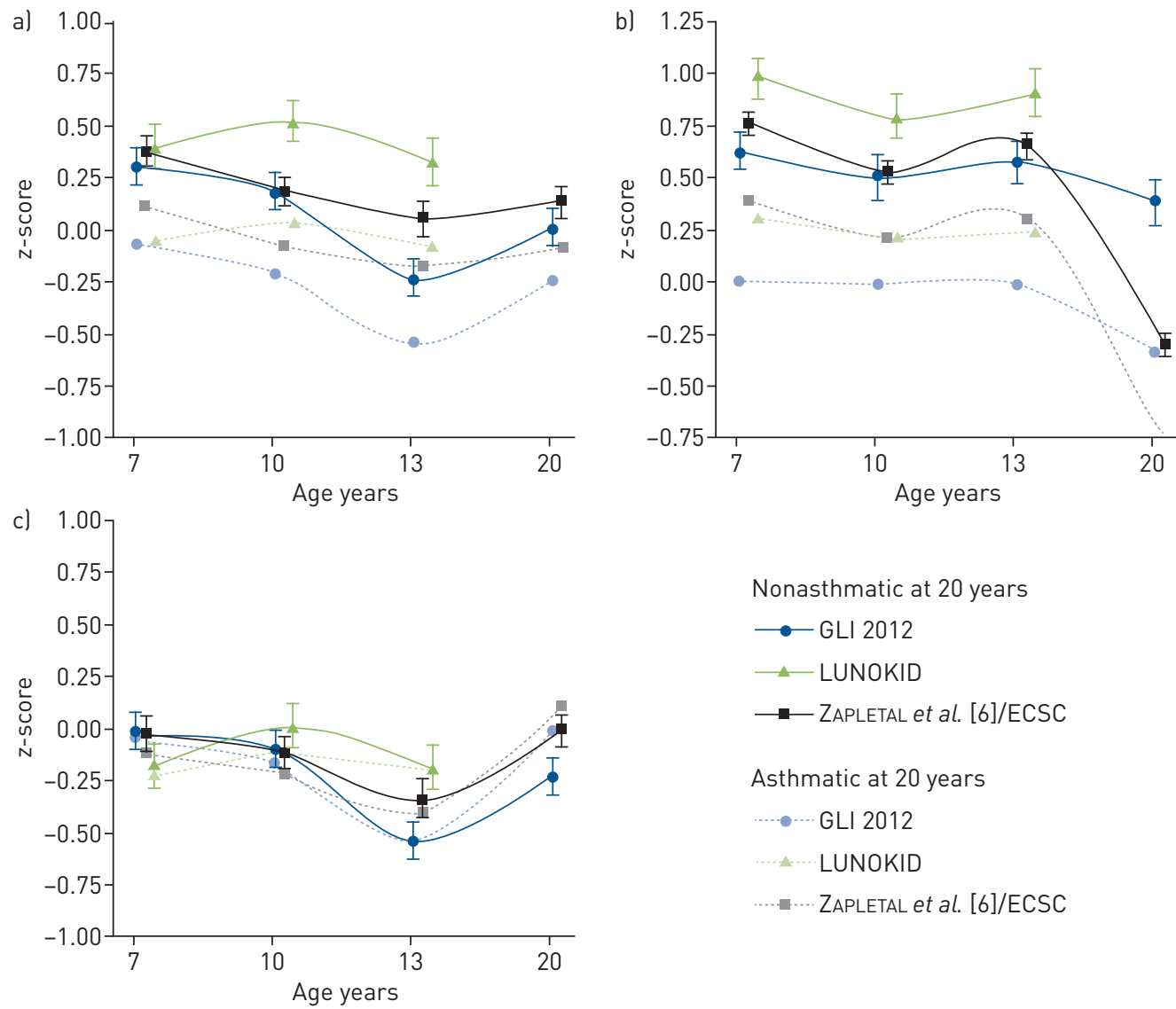

Asthmatic at 20 years

$\cdots$ GLI 2012

$\triangle \cdots$ LUNOKID

- ZAPLETAL et al. [6]/ECSC

FIGURE 1 Longitudinal development of a) forced expiratory volume in $1 \mathrm{~s}$ (FEV 1 ), b) FEV 1 /forced vital capacity (FVC) and c) FVC (mean z-scores and 95\% confidence intervals) by asthma status at age 20 years and by reference equation used (Global Lung Function Initiative (GLI) 2012, LUNOKID (Lungenfunktions-Normalwerte bei Kindern in Deutschland [Lung function Normal Values of Children in Germany]) and ZAPLETAL et al. [6]/ European Community for Steel and Coal (ECSC)). 
Previous studies suggested that additional parameters interfere with lung volume growth and lung function outcome in puberty, such as weight or puberty stage. NÈve et al. [11] looked at pubertal stage by Tanner scale and time since menarche, and found that thoracic and lung development occurred predominantly more towards the end of puberty in males and rather earlier in puberty in females. BEKKERs et al. [12] reported a significant relationship between body mass index and waist circumferences and FVC in children at 12 years of age. Other factors that influence lung growth in the transition from childhood to adulthood include bone age, growth velocity, stature, skeletal and muscle fat mass, and chest circumference [13]. However, none of these factors are included in reference equations.

We saw a difference in a lung volume decrease between boys and girls at the age of 13 years. This may be due to the fact that at this puberty age, lung development is still occurring in boys, whereas lung development is almost finished in girls. A similar observation was made in a cross-sectional study by HüLS et al. [14], applying GLI reference equations to original lung function data from LUNOKID and GINIplus (German Infant Study on the Influence of Nutrition Intervention plus Air pollution and Genetics on Allergy Development) at the age of 15 years. The authors underline that height development and therefore lung volume increase varies much between Caucasian populations, especially in puberty when growth in males is rapid. In our opinion, the differences are not only present between Caucasian populations but also between local populations and individuals.

Thus, results from our present study emphasise that lung function data, especially FEV1 and FVC should be interpreted with caution in the age group of 13 years, when pubertal cofactors might play role. Longitudinally, FEV1/FVC deviated least over time until puberty regardless of which reference equations were used. Using GLI reference equations for FEV1/FVC may even provide a reliable reference throughout to adulthood.

In our population-based birth cohort population, the application of GLI reference equations did not reveal a steady spline curve of FEV1, as shown for a cystic fibrosis patient population by STANOJEVIC et al. [4]. The reasons for that are unclear, and it may have been due to differences in the populations, subject numbers and/or the applied methods.

The classification of asthmatic and nonasthmatic subjects at the age of 20 years may have led to some misdiagnosis. However, the number of asthmatic subjects at 13 years who were not symptomatic at 20 years was small. Instead of a single birth cohort like MAS, combined multiple cohort datasets with increased statistical power are required to analyse subphenotypes such as persistent asthma, transient asthma and asthma in remission.

Considering the nonasthmatic group as a healthy group, the reference equations used seem to characterise lung function fairly well, as $\mathrm{z}$-scores of $\mathrm{FEV} 1$ and $\mathrm{FVC}$ remained within the normal thresholds of -0.5 to 0.5 . Interestingly, z-scores of FEV1/FVC were much larger than 0 regardless of which reference equations we used, showing less airway obstruction in this group compared to the reference population.

In summary, our results showed a drop in the FEV1 and FVC of both our nonasthmatic and asthmatic group at the age of 13 years regardless of which reference equations were used. Using FEV1/FVC values and applying them to the reference data of GLI or LUNOKID might be useful for longitudinal follow-up analysis but further evaluations in other settings are required.

Christiane Lex ${ }^{1}$, Marvin Reuter $\circledast^{2,3}$, Antje Schuster ${ }^{3}$, Linus Grabenhenrich ${ }^{4}$, Carl-Peter Bauer ${ }^{5}$, Ute Hoffmann ${ }^{5}$, Johannes Forster ${ }^{6}$, Fred Zepp ${ }^{7}$, Ulrich Wahn ${ }^{8}$, Thomas Keil ${ }^{9}$, Susanne Lau ${ }^{8}$ and Dirk Schramm ${ }^{3}$

${ }^{1}$ Dept of Paediatric Cardiology and Intensive Care Medicine with Paediatric Respiratory Medicine, University Medical Center Goettingen, Goettingen, Germany. ${ }^{2}$ Institute of Medical Sociology, Centre for Health and Society, Medical Faculty, University of Duesseldorf, Duesseldorf, Germany. ${ }^{3}$ Dept of Paediatrics, Heinrich-Heine-University, Duesseldorf, Germany. ${ }^{4}$ Dept for Infectious Disease Epidemiology, Robert Koch-Institut, Berlin, Germany. ${ }^{5}$ Dept of Pediatrics, Technical University of Munich, Munich, Germany. ${ }^{6}$ St Josef s Hospital, Dept of Pediatrics, Freiburg, Germany. ${ }^{7}$ Center for Pediatric and Adolescent Medicine, University Medical Center Mainz, Freiburg, Germany. ${ }^{8}$ Dept of Pediatric Pneumology and Immunology, Charité - Universitätsmedizin Berlin, Berlin, Germany. ${ }^{9}$ Institute for Social Medicine, Epidemiology and Health Economics, Charité - Universitätsmedizin Berlin, Berlin, Germany.

Correspondence: Christiane Lex, Dept of Paediatric Cardiology and Intensive Care Medicine with Paediatric Respiratory Medicine, University Medical Center Goettingen, Robert-Koch-Straße 40, 37075 Goettingen, Germany. E-mail: christiane.lex@med.uni-goettingen.de

Received: Feb 212018 | Accepted after revision: June 112018

Acknowledgements: Among all the collaborators over $>20$ years of MAS, the authors would especially like to thank Renate L. Bergmann and Christian E. Bergmann (Dept of Obstetrics, Charité - Universitätsmedizin Berlin, Berlin, Germany). The authors are also grateful to Ursula Krämer and Anke Hüls (Environmental Health Research Institute, Heinrich Heine University, Dusseldorf, Germany) for providing LUNOKID reference data. 
Conflict of interest: S. Lau has served on advisory boards for DBV, ALK and Boehringer, outside the submitted work. J. Forster reports receiving grants from Federal Ministry of Health during the conduct of the study.

Support statement: MAS was funded by grants from the German Federal Ministry of Education and Research (07015633, 07 ALE 27, 01EE9405/5 and 01EE9406) and the German Research Foundation (KE 1462/2-1). The funders had no role in the design, management, data collection, analysis or interpretation of the data or in the writing of the manuscript or the decision to submit for publication. Funding information for this article has been deposited with the Crossref Funder Registry.

\section{References}

1 Quanjer P, Stanojevic S, Cole T, et al. Multi-ethnic reference values for spirometry for the 3-95-yr age range: the global lung function 2012 equations. Eur Respir J 2012; 40: 1324-1343.

2 Hüls A, Krämer U, Gappa M, et al. New spirometric reference values for children and adolescents in Germany considering height and non-linear age effects: the LUNOKID-study. Pneumologie 2013; 3: 141-149.

3 Hüls A, Krämer U, Gappa M, et al. Erratum. New spirometric reference values for children and adolescents in germany considering height and non-linear age effects: the LUNOKID-study. Pneumologie 2014; 6: 393.

4 Stanojevic S, Bilton D, McDonald A, et al. Global Lung Function Initiative equations improve interpretation of FEV1 decline among patients with cystic fibrosis. Eur Respir J 2015; 46: 262-264.

5 Bergmann R, Bergmann K, Lau-Schadensdorf S, et al. Atopic diseases in infancy. The German Multicenter Atopy Study (MAS-90). Pediatr Allergy Immunol 1994; 5: 19-24.

6 Zapletal A, Samánek M, Paul T. Lung Function in Children and Adolescents (Progress in Respiration Research). Basel, Karger-Verlag, 1987.

7 Quanjer P, Tammeling G, Cotes J, et al. Lung volumes and forced ventilatory flows. Eur Respir J 1993; 6: Suppl. $16,5-40$.

8 Lødrup C, Carlsen KC, Roll S, et al. Does pet ownership in infancy lead to asthma or allergy at school age? Pooled analysis of individual participant data from 11 European birth cohorts. PLoS One 2012; 7: e43214.

9 Tischer CG, Hohmann C, Thiering E, et al. Meta-analysis of mould and dampness exposure on asthma and allergy in eight European birth cohorts: an ENRIECO initiative. Allergy 2011; 66: 1570-1579.

10 Hall G, Thompson B, Stanojevic S, et al. The Global Lung Initiative 2012 reference values reflect contemporary Australian spirometry. Respirology 2012; 17: 1150-1151.

11 Nève V, Girard F, Flahault A, et al. Lung and thorax development during adolescence. Relationship with pubertal status. Eur Respir J 2002; 20: 1292-1298.

12 Bekkers M, Wijga A, Gehring U, et al. BMI, waist circumference at 8 and 12 years of age and FVC and FEV1 at 12 years of age. The PIAMA birth cohort study. BMC Pulm Med 2015; 15: 39.

13 Lebowitz M, Sherrill D. The assessment and interpretation of spirometry during the transition from childhood to adulthood. Pediatr Pulmonol 1995; 19: 143-149.

14 Hüls A, Krämer U, Gappa M, et al. Age dependency of GLI reference values compared with paediatric lung function data in two German studies (GINIplus and LUNOKID). PLoS One 2016; 11: e0159678. 